\title{
Fomento del desarrollo del pensamiento crítico en estudiantes de grado décimo desde situaciones cotidianas en la asignatura de Filosofía*
}

\author{
José Miguel Lara Rodríguez ${ }^{1}$ \\ orcid.org/0000-0002-2462-8375 \\ Institución Educativa Distrital Raimundo Sojo, Barranquilla, Colombia \\ Elquis Rodríguez Guerra ${ }^{2}$ \\ orcid.org/0000-0002-0795-9703 \\ Fundación Universitaria Católica del Norte, Barranquilla, Colombia
}

DOI: http://dx.doi.org/10.17081/eduhum.18.31.1383

Recibido: 3 de marzo de 2016

Aceptado: 27 de agosto de 2016

\section{Promotion of critical thought for tenth grade students about everyday situations in the Philosophy class}

Palabras clave:

Pensamiento crítico,

Habilidades cognitivas,

Competencia,

Situaciones cotidianas, Filosofía.

Keywords:

Critical thought,

Research- action methodology,

Philosophy, Diagnostic instrument.

\begin{abstract}
Resumen
Este artículo presenta el fomento del desarrollo del pensamiento crítico en estudiantes de grado décimo B del Instituto de Educación Distrital Técnico Jesús Maestro Fe y Alegría de la ciudad de Barranquilla (Colombia), mediante la atención a situaciones cotidianas en la asignatura de filosofía, enmarcado en la implementación de la metodología investigación-acción. En esta investigación se encontró que los estudiantes tenían deficiencias de pensamiento crítico, evidenciado en temas propios de la asignatura de Filosofía. Se ideó fomentar el desarrollo del pensamiento crítico en los estudiantes atendiendo una situación cotidiana relacionada a un tema filosófico. Se aplicó un instrumento diagnóstico que evaluó las habilidades de: interpretación, análisis y explicación (Facione, 1990), relacionadas a una situación cotidiana, que arrojó resultados con nivel bajo. Posteriormente, se aplicaron estrategias para mejorar estas habilidades y, en general, el problema detectado. Finalmente se ejecutó un instrumento diagnóstico, similar al inicial, que evidenció mejoría en los estudiantes, pasando de un nivel bajo a básico.
\end{abstract}

\begin{abstract}
This article promotes the development of critical thought in tenth (B) grade students of the District Institute of Education "Jesus Maestro", managed by "Fe y Alegría" Barranquilla (Colombia). According to everyday situations in the subject of Philosophy, the work has implemented the research-action methodology. This research work has found some deficiencies in students when looking at critical thought, which has become more evident in topics that refer to Philosophy. The main idea was to foster critical thought on students according to everyday situations related to topics about philosophy. A diagnostic instrument was applied in order to evaluate skills: interpretation, analysis, and explanation, related to an everyday situation, whose results reached a lower level. Further on, some strategies were applied to improve these skills, and, in general, the problem was detected. Finally, a diagnostic instrument was used, similar to the first one, which evidenced an improvement on students, as they passed from a Low level to an upper Basic level.
\end{abstract}

\section{(c) $(9)$}

Referencia de este artículo (APA): Lara, J. M. \& Rodríguez, E. (2016). Fomento del desarrollo del pensamiento crítico en estudiantes de grado décimo desde situaciones cotidianas en la asignatura de Filosofía. Revista Educación y Humanismo, 18(31), 343-357. http://dx.doi.org/10.17081/eduhum.18.31.1383

\footnotetext{
* Este artículo fue extraído del proyecto de grado titulado: Fomento del desarrollo del pensamiento crítico en estudiantes de grado décimo B del Instituto de Educación Distrital Técnico Jesús Maestro Fe y Alegría de Barranquilla mediante la atención a situaciones cotidianas en la asignatura de filosofía. Dicho proyecto fue realizado en el marco de las prácticas pedagógicas, que exige la Fundación Universitaria Católica del Norte, y aprobado por la misma para a otorgar el título de: licenciado en Filosofía y Educación Religiosa.

1. Licenciado en Filosofía y Educación Religiosa, Institución Educativa Distrital Raimundo Sojo. Email: lara73754@gmail.com

2. Licenciado en Filosofía y Ciencias Religiosas y Magíster en Ética Social y Desarrollo Humano, Fundación Universitaria Católica del Norte. Email: erodriguezg@ucn.edu.co
} 


\section{Introducción}

La investigación fue realizada en el contexto de las prácticas pedagógicas en la Institución Educativa Distrital Jesús Maestro, Fe y Alegría. Este plantel educativo está ubicado al suroccidente de la ciudad de Barranquilla (Colombia), y la población utilizada estuvo constituida por 31 estudiantes del curso $10^{\circ} \mathrm{B}$. Este trabajo se caracteriza por ser de enfoque cualitativo; tipo de investigación que es ideográfica (datos textuales detallados, descriptivos), encuentra nociones, ideas compartidas dando sentido al comportamiento social, se ejercita con la palabra, argumentos, supuestos, imágenes visuales, gestos, representaciones, indicios de cultura material, asimila y presenta sus comprensiones en textos escritos, videos, o audiovisuales (Galeano, 2004). Se implementó también la metodología investigación-acción, como parte de la investigación cualitativa y que según Bisquerra (2009) se puede definir como: "una reflexión sobre las acciones humanas y las situaciones sociales vividas por los profesores, que tiene como objeto ampliar la comprensión (diagnostico) del profesor y de sus problemas prácticos" (Bisquerra, 2009, p.370).

Los instrumentos diagnósticos y las actividades didácticas tienen en común la relación de una reflexión filosófica a una situación cotidiana, con la que se ejercita el pensamiento crítico aplicando habilidades cognitivas, también están enmarcados en el concepto de pensamiento crítico que se construyó, el cual fusiona los enfoques de los autores como Campos (2007), que confirma y enfatiza el pensamiento crítico hacia el uso de habilidades cognitivas, Facione (1990) que enfatiza el pensamiento crítico hacia el juicio deliberado y autorregulado y Chance (1986), que menciona como cualidad del pensamiento crítico el análisis de la realidad. Los tres conceptos de los mencionados autores, coinciden en el uso de habilidades cognitivas como evidencia de pensamiento crítico. Las habilidades cognitivas se tomaron de Facione (1990): interpretación, análisis y explicación, ya que facilita muchos ejemplos para el desempeño de estas habilidades, y sub habilidades que permitieron la creación de indicadores de logros para la evaluación de los estudiantes. Se notó también que las explicaciones, apuntan a que estas habilidades se mejoran con estrategias propias del constructivismo, al igual que otras sub habilidades que propuso el autor, pero que no se tuvieron en cuenta para este trabajo. También se establecieron juicios valorativos para el desempeño de cada sub habilidad escogida, como también para el nivel total de pensamiento crítico, estos juicios corresponden a la escala de valoración nacional: bajo, básico, alto y superior (Ministerio de Educación Nacional, [MEN], 2009), que permiten en la educación primaria, básica y media, determinar los niveles de rendimiento de los estudiantes. Finalmente, las actividades didácticas utilizan como situaciones cotidianas: los videos y los textos.

\section{Problema de investigación}

Este trabajo fue consecuencia de la problemática que se percibía en las prácticas pedagógicas cuando los alumnos mostraban deficiencias 
al responder preguntas en los talleres y evaluaciones de temas propios de la asignatura de Filosofía, que exigían pensar críticamente mediante habilidades cognitivas. La anterior situación requirió ejercitarlos en una estrategia que fomentara el desarrollo del pensamiento crítico con las sub habilidades propuestas por Facione (1990), que ayudan a desarrollar dicha competencia. Se escogieron posteriormente las situaciones cotidianas como un medio ideal para el fomento del desarrollo del pensamiento crítico, como así lo menciona el Ministerio de Educación Nacional (2010) al definirse como:

Fruto de un trabajo riguroso, validado con la comunidad académica y educativa a nivel nacional, en acuerdo con la idea de que las nuevas generaciones interioricen y pongan en práctica en su vida diaria el ejercicio de la reflexión filosófica" (p.9).

Hecho que lleva a plantear la siguiente pregunta de investigación: ¿cómo se puede fortalecer el pensamiento crítico en estudiantes de grado décimo $\mathrm{B}$ del Instituto de Educación Distrital Técnico Jesús Maestro, Fe y Alegría de Barranquilla mediante la atención a situaciones cotidianas en la asignatura de filosofía?

Para este estudio se decidió que las estrategias implementadas en el aula relacionarán un tema filosófico con una situación cotidiana, de tal forma que los estudiantes se ejercitasen en el pensamiento crítico, aplicando las determinadas habilidades cognitivas que se decidieron trabajar.
Lo anterior encontró sentido en la idea de que, el hombre vive diversas situaciones cotidianas que exigen afrontarlas con reflexión, por lo tanto el trabajo para desarrollar el pensamiento crítico en los procesos formativos, toma considerable importancia, como lo menciona el Ministerio de Educación Nacional (2010):

El pensamiento crítico se evidencia en el examen de las posturas ajenas pero también en el de las propias, por lo que contribuye en el desarrollo de la autocrítica necesaria para tomar distancia de las propias posiciones y de los marcos paradigmáticos desde donde ellas son formuladas (p.31).

En la actualidad el sistema educativo colombiano, no es ajeno a la idea que: “el estudio, comprensión y uso del pensamiento crítico no sólo es importante, sino también necesario en todos los niveles educativos" (Campos, 2007, p.9).

\section{Diagnóstico del nivel de pensamiento crí-} tico de los estudiantes de grado decimo $\mathrm{B}$ del Instituto de Educación Distrital Técnico Jesús Maestro, Fe y Alegría

\subsection{Pensamiento crítico}

Existen múltiples enfoques de conceptos sobre pensamiento crítico, para el desarrollo de esta investigación se tuvieron en cuenta algunos de ellos.

Varios estudiosos como Facione (2007) y Lipman (1997) han enfocado el concepto de pensamiento crítico dándole relevancia al juicio. El 
juicio, es deliberado y también autorregulado, se puede aplicar al realizar interpretaciones, análisis o inferencias y así mismo en la capacidad de dar consideraciones de tipo: conceptuales, metodológicas de criterios, de evidencias y contextuales en las que se basa dicho juicio generado (Facione, 1990).

El uso de habilidades intelectuales es un enfoque válido del concepto de pensamiento crítico, precisamente Rioseco y Ziliani (1998), Campos (2007), Lombardero (2015) y Fowler (1996), consideraron en sus investigaciones lo anteriormente comentado. Combinar de manera compleja habilidades intelectuales con fines determinados, ya sea analizando cuidadosa y lógicamente información para determinar su validez o la veracidad de la argumentación o premisas que esta implique, y la solución de problemas, son indicios de pensamiento crítico, a dicha idea se adiciona que pensar críticamente es también pensar claro y racionalmente, favoreciendo así al desarrollo del pensamiento reflexivo e independiente, lo que permite tener juicios confiables de lo que se cree de una afirmación o de la conveniencia de una determinada acción (Campos, 2007).

Evaluar el pensamiento es uno de los puntos de vista que ha enmarcado la definición de pensamiento crítico, este aspecto fue tratadopor ejemplo, en las investigaciones de Chaffee (1990) y Richard y Elder (2005). El pensamiento crítico requiere analizar y valorar el pensamiento para mejorarlo, es necesario entonces conocer las estructuras más básicas del pensamiento, elementos y estándares, ya que llegar a la verdadera mejoría del pensamiento implica reestructurarlo, esto como resultado de un efectivo análisis y evaluación del mismo.

Otro enfoque del concepto de pensamiento crítico está en los que implican evaluación de la realidad, en este sentido, Chance (1986) conceptúa el pensamiento crítico como: "la habilidad de analizar hechos, generar y organizar ideas, defender opiniones, realizar comparaciones, derivar inferencias, evaluar argumentos y resolver problemas" (p.6), lo que se asume en este trabajo. Importante es que el alumnado se concientice de la actitud del análisis de la realidad, lo cual implica atención a su diario vivir; aspecto considerado de vital importancia para la asignatura de Filosofía y su valor de filosofar en la realidad (MEN, 2010).

\section{Método}

2.1. Proceso del diagnóstico inicial del nivel del pensamiento crítico

Para el diagnóstico del pensamiento crítico se tuvo en cuenta los siguientes pasos:

Se construyó el concepto de pensamiento crítico incorporando las definiciones de Campos (2007), Facione (1990) y Chance (1986), ya que coinciden en dar a entender la idea de que pensar críticamente implica el uso de ciertas habilidades cognitivas, y porque se tuvo en cuenta también para dicha construcción, las particularidades de: el uso del juicio deliberado y autorre- 
gulado de Facione (1990), la habilidad de análisis de la realidad de Chance (1986) y el énfasis en pensamiento crítico en el uso de habilidades cognitivas de Campos (2007).

Al concepto construido se enlazó las sub habilidades de interpretación, análisis y explicación de Facione (2007), ya que éste da muchos ejemplos para el desempeño de estas habilidades y prodiga sub habilidades que permitieron la creación de indicadores de logros para la evaluación; además las explicaciones del autor apuntan a que estas habilidades se mejoran con estrategias propias del constructivismo. Se escogieron luego, sub habilidades que sirvieron para la elección de indicadores.

Se diseñó el instrumento diagnóstico compuesto por una reflexión filosófica, una lectura o video que implica una situación cotidiana relacionada a dicha reflexión, y tres ejercicios, que con base a dicha situación cotidiana, pretenden evaluar cada uno, al estudiante en determinada habilidad del pensamiento crítico y su respectivos indicadores de logros*.

Se le aplicó 1 instrumento diagnóstico (30 minutos para responder) a la población constituida, la cual generó resultados ubicados en un nivel bajo, lo que se describirá posteriormente.

* Ejercicio 1: evalúa la habilidad interpretación; ejercicio 2: evalúa la habilidad análisis; ejercicio 3 : evalúa la habilidad explicación. Nota: En la habilidad interpretación el alumno debe responder dos pregunta (subhabilidades) equivalentes cada una a 0,5 y que sumadas dan un total de 1 ; en habilidad análisis responderá una pregunta que equivale a 1 ; y en la habilidad argumentación deberá presentar 4 razones donde cada una equivale a 0,25 y que sumadas equivalen a 1 .
Tabla 1. Construcción del concepto de pensamiento crítico

\begin{tabular}{|c|c|c|}
\hline \multicolumn{3}{|c|}{ Pensamiento Crítico } \\
\hline Chance (1986) & \multicolumn{2}{|c|}{$\begin{array}{l}\text { "la habilidad de analizar hechos, generar y } \\
\text { organizar ideas, defender opiniones, realzar } \\
\text { comparaciones, derivar inferencias, evaluar } \\
\text { argumentos y resolver problemas" (p.6). }\end{array}$} \\
\hline Facione (1990) & \multicolumn{2}{|c|}{$\begin{array}{l}\text { "es el juicio deliberado y autorregulado, } \\
\text { que se usa para interpretar, analizar e in- } \\
\text { ferir, así como para explicar las conside- } \\
\text { raciones conceptuales, metodológicas, de } \\
\text { criterio, de evidencias y contextuales en las } \\
\text { cuales se basa el juicio dado" (p.20). }\end{array}$} \\
\hline Campos (2007) & \multicolumn{2}{|c|}{$\begin{array}{l}\text { "como una combinación compleja de ha- } \\
\text { bilidades intelectuales que se usa con fines } \\
\text { determinados, entre ellos el de analizar } \\
\text { cuidadosa y lógicamente información para } \\
\text { determinar su validez, la veracidad de su } \\
\text { argumentación o premisas y la solución de } \\
\text { una problemática. El pensamiento crítico es } \\
\text { el pensar claro y racional que favorece el } \\
\text { desarrollo de pensamiento reflexivo e inde- } \\
\text { pendiente que permite a toda persona reali- } \\
\text { zar juicios confiables sobre la credibilidad } \\
\text { de una afirmación o la conveniencia de una } \\
\text { determinada acción" (p.19). }\end{array}$} \\
\hline $\begin{array}{c}\text { Habilidad del } \\
\text { Pensamiento } \\
\text { Crítico } \\
\end{array}$ & $\begin{array}{c}\text { Sub } \\
\text { Habilidad }\end{array}$ & Indicador \\
\hline \multirow[t]{2}{*}{ La interpretación } & $\begin{array}{l}\text { Decodificación } \\
\text { del significado }\end{array}$ & $\begin{array}{l}\text { identifica una intención } \\
\text { no enunciada en el texto. }\end{array}$ \\
\hline & $\begin{array}{l}\text { Aclarar } \\
\text { significancia }\end{array}$ & $\begin{array}{l}\text { Reconoce el significa- } \\
\text { do de expresiones en un } \\
\text { texto. }\end{array}$ \\
\hline El análisis & $\begin{array}{l}\text { Analizar } \\
\text { argumentos }\end{array}$ & $\begin{array}{l}\text { Identifica un juicio no } \\
\text { enunciado basado en el } \\
\text { estudio profundo de una } \\
\text { situación. }\end{array}$ \\
\hline La explicación & $\begin{array}{l}\text { Presentar } \\
\text { argumentos }\end{array}$ & $\begin{array}{l}\text { Presenta argumentos } \\
\text { completos y bien razo- } \\
\text { nados en contexto de evi- } \\
\text { denciar comprensión. }\end{array}$ \\
\hline
\end{tabular}

Fuente: Elaboración propia a partir de Chance (1986), Facione (1990) y Campos (2007) 
Paralelamente a lo anterior, se diseñó las siguientes equivalencias y escala de valores.

Habilidades: interpretación, análisis, explicación: cada habilidad se rige por la siguiente escala de valores: valor mínimo $=0$, valor máxi$\mathrm{mo}=1$; entre 0,0 y $0,5=$ bajo, entre 0,6 y 0,7 $=$ básico, entre 0,8 y $0,9=$ alto, $1.0=$ superior. PENSAMIENTO CRITICO es igual a la suma de los puntos arrojados por las habilidades interpreta, analiza y explica*, siendo su valor Máximo 3 y su valor Mínimo igual a 0 ; seguidamente para este concepto se diseñó la siguiente escala de valores: entre 0,0 y $1,7=$ bajo, entre 1,8 y 2,2 $=$ Básico, entre 2,3 y 2,7 = alto, y entre 2,8 y 3,0 $=$ Superior.

2.2. Descripción del nivel de pensamiento crítico del primer diagnóstico por debajo de la mitad de su rendimiento máximo. En los resultados: 0,3709677 sobre 0,5 para aclaración del sentido y 0.112903226 sobre 0,5 para decodificación del significado (sub habilidades), se evidencia en la primera un mejor desempeño. La habilidad interpretación fue la que tuvo mejor desempeño. La habilidad análisis muestra un promedio de 0.419355 , lo cual denota el rendimiento bajo, ya que esta habilidad sólo tiene una sub habilidad (analizar argumentos), dándonos una idea de cómo los estudiantes se encuentran en su capacidad de identificar un juicio no enunciado basado en el estudio profundo de una situación; el resultado de esta habilidad ubica a los estudiantes en un nivel bajo, según escala. Para la habilidad Explicar cuya sub habilidad estudiada es presentar argumentos, y cuyo indicador es: presenta argumentos completos y bien razonados en contexto de evidenciar com-

Tabla 2. Descripción inicial del nivel del pensamiento crítico

\begin{tabular}{|l|c|c|c|c|c|}
\hline \multirow{2}{*}{} & \multicolumn{2}{|c|}{ Interpreta } & Analiza & Explica & Pensamiento Crítico \\
\cline { 2 - 5 } & SUBHAB 1 & SUBHAB 2 & SUBHAB 3 & SUBHAB 4 & TOTAL P. CRíTICO \\
\hline Promedio Subtotal Interpreta & 0,112903226 & 0,3709677 & & & \\
\hline Promedio Total Habilidades & 0,483870968 & 0,419355 & 0,387096774 & \\
\hline Promedio Pensamiento Crítico & \multicolumn{2}{|l|}{} & 1,290322581 \\
\hline
\end{tabular}

Fuente: Resultados de investigación

La anterior tabla evidencia que la interpretación obtuvo un promedio de 0,483870968 , sobre uno (1) mostrando a los estudiantes levemente

* En la evaluación diagnostica para producir los puntos arrojados por determinada habilidad, se va sumando los puntos que cada estudiante evaluado obtuvo en dicha habilidad y se divide por el número total de estudiantes evaluados, es decir, cada habilidad es un promedio. El pensamiento crítico es la suma dichos promedios (habilidades). prensión, muestra el más bajo de los promedios obteniendo solo 0.387096774 situándolos en un nivel bajo, siendo la habilidad de mayor dificultad para la población. Seguidamente la suma de los promedios individuales obtiene una puntuación de 1.290322581, ubicándose dentro del rango del nivel bajo, según la escala diseñada para determinar el nivel de pensamiento crítico. 
2.3. Estrategias para el mejoramiento del pensamiento crítico

Se tuvo en cuenta que, "existe más de una manera o forma de realizar el pensamiento crítico" (Campos, 2007, p.63), exponiéndose a continuación algunos ejemplos. La técnica más simple es el uso de preguntas: que generen puntos de vista, solución, etc., utilizando la discusión grupal, el dialogo controversial, entre otros (Campos, 2007); otro ejemplo sería el organizar gráficamente un tema de la manera como lo considere el alumno, transmitiendo el mismo mensaje (Facione, 2007); también el aprendizaje basado en problemas que genera en los alumnos el ánimo en procedimientos propios de investigación (Barrel, 1999); por último se puede mencionar la técnica del ensayo, ya que ésta posee un discurso con lenguaje académico y no coloquial, con unidad argumentativa que implica dar razón de lo que se niega y afirma (Guajardo \& Serrano, 2001).

A continuación se realiza una descripción de las estrategias utilizadas en esta investigación.

La primera actividad fue didáctica titulada: "dialogo interactivo", y que tiene como objetivo: concientizar a los estudiantes de la importancia de mantenerse bien informados, como punto de partida para el fomento del pensamiento crítico. $\mathrm{Su}$ desarrollo inició con la explicación de unos aportes sobre política del pensador y político Tomás Moro (2013) como: el poder absoluto, la ambición de riquezas y la desigualdad económica, también se tomaron ideas del pensador Ni- colás Maquiavelo (1513) como: la adquisición, conservación y aumento del poder, y la política como labor no apta para santos; seguidamente se explicó de manera breve conceptos relacionados con el sistema de salud colombiano; después se proyectaron fragmentos claves de un video que donde se explicaban anomalías del sistema de salud de Colombia, Prieto (2013), y se procedió a confrontar los aportes de los anteriores filósofos notados en el video, así como también aclarar algunas situaciones del video gracias a los conceptos explicados; finalmente se hizo un dialogo con los estudiantes sobre pensamiento crítico. El tiempo de realización fue de una hora y media.

La segunda actividad estratégica fue titulada: "piensa en tu educación", tiene como objetivo: ejercitar al alumno en la relación de conceptos, mapa conceptual y el ensayo. Su desarrollo implicó: la explicación de unos aportes sobre educación del filósofo Immanuel Kant (1803) como: una educación bien razonada, las escuelas dirigidas por gente idónea, hombres educadores sin una buena educación, y también se tomaron ideas del también filósofo Jean-Jacques Rousseau (1762) como: la educación que suele ser alienante, y la necesidad de educar acorde a las necesidades; posteriormente los alumnos leyeron un artículo que hablaba de los motivos de una deficiente educación en Colombia, del autor Gossaín (2014), y se confrontaron los aportes de los filósofos mencionados con la lectura; seguidamente los alumnos, con base al artículo, realizaron ejercicios para desarrollar el pensamiento 
crítico (relación de concepto, mapa conceptual, ensayo). El tiempo de realización: fue de una hora y media.

La tercera actividad fue titulada: "ser críticos y no alienados", tiene como objetivo: ejercitar al estudiante en la selección de las ideas centrales de un tema, ejercitar su capacidad dialógica, y parafrasear. Su desarrollo implicó: la explicación de algunos aportes de Karl Marx (1975) sobre la alienación; se colocó algunos fragmentos de un video que explica la injusticia a los tradicionales agricultores colombianos, obligados a comprar un producto denominado: semillas certificadas (Solano, 2013), confrontándolo con las ideas de Marx; esto ayudó a ejercitar a los estudiantes, con base al video, en la selección de ideas centrales, dialogo sobre puntos en común y en contra de dicha selección y el parafraseo de la idea de Marx (1975) "los productos de la mente humana parecen figuras autónomas, dotadas de vida propia, en relación unas con otras y con los hombres. Otro tanto ocurre en el mundo de las mercancías con los productos de la mano humana" (p.1030).

La cuarta actividad fue titulada: "críticos en la fe", tiene como objetivo: ejercitar a los alumnos en mapa conceptual y argumentación a través de la opinión. Su desarrollo implicó: la explicación de la crítica de Marx (1844) sobre la religión como medio en que el hombre tiende a no pertenecerse, actuando la fantasía humana; los estudiantes leyeron el artículo, que explica cómo la fe en Colombia se convierte en un mercado
(Merchán, 2013), y se confrontó con las citadas reflexión de Marx; posteriormente, con base al artículo, los alumnos se ejercitaron en completar un mapa conceptual, y la argumentación a través de la opinión sobre el tema de la libertad de culto en Colombia. El tiempo de realización fue de una hora y media.

La quinta actividad estratégica fue titulada: “críticos por la paz", tiene como objetivo: ejercitar a los estudiantes en la importancia del conocimiento de conceptos para mayor comprensión de un tema y la argumentación a través del ensayo. En su desarrollo se explicaron aportes de Thomas Hobbes (1651) sobre la persona humana como naturalmente antisocial y con sed permanente de poder; los estudiantes vieron un fragmento de un video que muestra la problemática de pandillas en la ciudad de Barranquilla (García, 2013); posteriormente los estudiantes, con base a las anteriores fuentes informativas, se ejercitaron en: en mapa mental y ensayo. El tiempo de realización fue de una hora y media.

La sexta actividad fue titulada: "piensa en la vida", tiene como objetivo: ejercitar a los estudiantes en la identificación de ideas principales, secundarias, y argumentación a través del apoyo y rechazo de puntos de vista. En su desarrollo se explicaron ideas de Jean-Paul Sartre (1989) sobre el hombre como ser libre; se leyó un artículo que explica la dificultad constitucional con relación al aborto (Reyes, 2015); se confrontó con los aportes de Sartre; luego los alumnos realizaron como actividad, seleccionar la ideas prin- 
cipales del artículo y cuatro ideas secundarias, más argumentar por qué apoyarían la frase: "No queremos más mujeres perseguidas ni juzgadas por hacer valer sus derechos" (Reyes, 2015, p.4), extraída del artículo usado en esta actividad. El tiempo de realización fue de una hora y media.

\subsection{Progresos del pensamiento crítico de los} estudiantes de grado decimo $B$ en el desarrollo de la investigación

En esta instancia se diseñó y aplicó un instrumento diagnostico final (30 minutos para responder), el cual está estructurado de la misma manera que el instrumento diagnóstico inicial, es decir, se relaciona una reflexión filosófica con un tema cotidiano; posee el mismo número de preguntas con el mismo puntaje a obtener en cada una. Las preguntas apuntan a verificar la destreza de pensamiento crítico del estudiante según la habilidad trabajada en determinada pregunta y según el indicador que le corresponda, etc. para aclaración del sentido y 0,306451613 sobre 0,5 para decodificación del significado, mostrando un desempeño más parejo que en el diagnóstico pasado. La habilidad interpretación obtuvo un rendimiento medio en comparación con las otras habilidades. La habilidad análisis muestra un promedio de 0,612903 sobre 1, lo cual denota que con relación al diagnóstico inicial muestra mejoría, pero que del nivel bajo no avanzó, siendo la puntuación más baja, evidenciando mayor dificultad para los estudiantes en la subhabilidad de analizar argumentos, y su capacidad de identificar un juicio no enunciado basado en el estudio profundo de una situación. Sobre la habilidad explicar cuya sub habilidad estudiada es presentar argumentos, y cuyo indicador es, el estudiantepresenta argumentos completos y bien razonados en contexto de evidenciar comprensión, se muestra el más alto rendimiento al obtener un promedio de 0,846774194 , ubicándose

Tabla 3. Descripción final del nivel del pensamiento crítico

\begin{tabular}{lccccc}
\hline & \multicolumn{2}{c}{ Interpreta } & Analiza & Explica & Pensamiento Crítico \\
& SUBHAB 1 & SUBHAB 2 & SUBHAB 3 & SUBHAB 4 & TOTAL P. CRÍTICO \\
\hline Promedio Subtotal Interpreta & 0,306451613 & 0,4516129 & & & \\
Promedio Total Habilidades & 0,758064516 & & 0,612903 & 0,846774194 & \\
Promedio Pensamiento Crítico & & & & & 2,169354839 \\
\hline
\end{tabular}

Fuente: Resultados de investigación

La tabla 3, evidencia que la interpretación dio un promedio de 0,758064516 , sobre uno (1) mostrando a los estudiantes por encima de la mitad de su rendimiento máximo, ubicándolos en un nivel básico en esta habilidad. Las dos sub habilidades no difieren tanto: 0,4516129 sobre 0,5 en un nivel alto. Posteriormente la suma de los promedios individuales arroja una puntuación de 2,169354839 , que ubica el crecimiento y rendimiento del pensamiento crítico en un nivel básico, aproximándose casi al nivel medio, según la escala diseñada. 


\section{Resultados y discusión}

En la presente instancia esta investigación mostró un avance de nivel bajo hacia básico, en cuanto al pensamiento crítico de los estudiantes, luego de implementar estrategias basadas en el uso de situaciones cotidianas mediante talleres que propendían por el desarrollo de las habilidades elegidas. La siguiente tabla nos muestra una comparación entre el estado inicial de los estudiantes y el estado final.

Tabla 4. Evolución del nivel de pensamiento crítico

Nivel del pensamiento crítico de los estudiantes de décimo grado del I.E.D. Técnico Jesús Maestro, Fe y Alegría

\begin{tabular}{|l|c|c|}
\hline & Resultados & Nivel \\
\hline Diagnóstico inicial & 1,290322581 & Bajo \\
\hline Diagnóstico final & 2,169354839 & Básico \\
\hline
\end{tabular}

Fuente: Resultados de investigación

Gráfico 1. Resultados inicial y final de la competencia pensamiento crítico

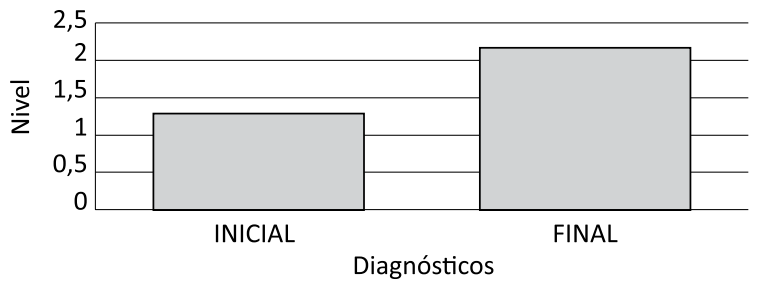

Fuente: Resultados de investigación

El trabajo requirió que, luego de la implementación de una prueba diagnóstica, en la que después de un proceso profundo de evaluación se obtuvo una valoración promedio de 1,290322581 sobre 3,0 , se diseñaran un grupo de actividades y talleres que ayudaran a mejorar las condiciones de pensamiento crítico de la población objeto de estudio.
El primer diagnóstico, utilizó tres habilidades mencionadas por Facione (1990) en su trabajo, que permitían determinar en qué estado se encontraban los estudiantes con respecto a su capacidad de pensar críticamente.

El diseño de las actividades y talleres que conllevarían a los estudiantes a mejorar su pensamiento crítico, pasó por un proceso de indagación que permitió conocer las características básicas esenciales de una actividad, para que permitiera el avance en las habilidades del pensamiento de una persona. Adicionalmente se buscaron temáticas que fuesen del ámbito cotidiano $\mathrm{y}$ que permitieran a los estudiantes mantenerse interesados en el desarrollo de las temáticas.

En este sentido, al revisar los resultados de la evaluación final 2,169354839 sobre 3,0, se puede observar un margen de diferencia que permite afirmar, que la implementación de la idea inicial ha sido satisfactoria; para evitar sesgos el instrumento de la evaluación final fue diseñado en la misma modalidad que la prueba diagnóstica.

Si bien es claro que el valor del aumento es de 0,96 décimas y que para alcanzar el grado superior aún se está lejos, los resultados permiten determinar posteriores de trabajos de investigación.

Desde la perspectiva teórica cabe mencionar que posterior a la revisión de las características necesarias para verificar la estructura del pensamiento crítico en un ser humano, se observó 
que existían una serie de habilidades y subhabilidades mentales que permiten observar sus características, por tanto el diseño de los ejercicios, tanto el de diagnóstico como los ulteriores, apuntaron a este grupo de habilidades señaladas por Facione (1990).

El resultado muestra un acercamiento a lo que se pretendía, que era mejorar las condiciones de pensamiento crítico a través del uso de situaciones cotidianas en la asignatura de Filosofía. La selección de las situaciones cotidianas es un punto especial dentro del trabajo, ya que estas deben responder a temáticas que despertarán en los estudiantes un interés mayor del que normalmente tienen en el desarrollo de la asignatura.

La conjugación de las categorías y teorías del pensamiento crítico en la práctica, son las que conllevaron a la obtención de estos resultados, y los que muy probablemente permitirán que la investigación se convierta en un aporte dentro del campo de los trabajos relacionados con el desarrollo del pensamiento crítico.

Si bien es claro que todas las habilidades y sub habilidades puestas en estudio tuvieron una mejoría entre el diagnóstico y la evaluación final, no todas mejoraron en el mismo porcentaje, sin embargo esto no es trascendental, ya que para efectos del resultado del trabajo, lo importante es el promedio final de la población, lo que muestra que los jóvenes fueron capaces de ser más atentos a las clases, modificar la forma como interpretaban, analizaban y explicaban situaciones a las que son expuestos diariamente y que en muchos casos requieren de una participación activa de ellos dentro de una comunidad, que le exige a los seres humanos convertirse en protagonistas de su proyecto de vida sin esperar que sean otros actores los responsables de su cambio.

El hecho de que las clases que utilizaron las estrategias didácticas, generaran en los estudiantes más atención y gusto que las clases que recibían de costumbre (explicación estricta de los temas filosóficos), permitió una mejor comprensión y sentido de las características del pensador crítico explicadas a ellos. Lo anterior recuerda que las características del pensador crítico hacen parte de la formación de esta competencia, cuestión que tendrá más fluidez y vivencia con clases amenas y significativas, producto del esfuerzo y dedicación del docente.

\section{Recomendaciones}

Después de verificar los resultados de este trabajo se recomienda que:

La formación para el pensamiento crítico debe ser un trabajo conjunto entre todos los docentes de la institución, ya que así puede adquirirse un crecimiento uniforme de esta competencia en todas las asignaturas y niveles educativos.

La institución, con relación a la formación del pensamiento crítico, debe enfocarse en teorías selectas basadas en el desarrollo de habilidades cognitivas (competencias), pues coincide con la manera de formar en Colombia al estudiantado en los niveles primaria básica y media. 
Se requiere que las clases de filosofía relacionen sus temas con situaciones cotidianas o también a otro tipo de circunstancia basadas en la realidad, lo que produce gusto y atención a los jóvenes por la materia, y permite que los alumnos puedan comprender y asimilar de manera significativa la teoría propia de esta asignatura, la cual suele ser compleja. En este sentido, el uso de recursos interactivos debe ser notablemente asiduo, no sólo a través del desarrollo de las clases en el aula, sino también en las actividades realizadas por los estudiantes en casa, es decir, que talleres y todo tipo de tareas incluyan la observación de videos, películas, que contengan situaciones cotidianas y reales para un mejor desarrollo de estos tipos de actividades.

El uso de situaciones cotidianas para la comprensión de los temas propios de la asignatura de Filosofía, apunta a recomendar que es menester para las entidades gubernamentales usar los avances en el campo de las investigaciones en pensamiento crítico, para orientar la construcción de filosofías de trabajo con los estudiantes de todos los niveles educativos, con el fin de generar caminos de productividad social, y de reencuentro con soluciones a problemas de la vida cotidiana en la que los ciudadanos no se sienten a esperar que las entidades del gobiernos sean los únicos protagonistas.

De igual manera, el cuerpo docente debe realizarse una autoevaluación sobre las estrategias que se implementan en la consecución de progresos de los estudiantes en el campo del desarrollo de las competencias básicas, y, si es necesario, realizar recomendaciones para las universidades que son al fin y al cabo las responsables de la formación de los docentes.

\section{Conclusiones}

Este trabajo cumplió con su cometido ya que la población de estudiantes presentó en un principio un rendimiento bajo, según escala implementada en la habilidad del pensamiento crítico (diagnóstico inicial), y después de las actividades didácticas aplicadas para la mejoría de la dificultad, el rendimiento de los estudiantes pasó a un nivel básico aproximándose casi a un nivel alto.

Las situaciones cotidianas relacionadas a temas propios de la asignatura de filosofía, son un medio adecuado para fomentar el desarrollo del pensamiento crítico; esto implica aplicar a dicha situación cotidiana habilidades que signifiquen destreza en pensamiento crítico.

Los avances mostrados por los estudiantes son una clara prueba de que estrategias bien diseñadas, y coordinadas con un buen modelo y paradigma metodológico, pueden direccionar las capacidades natas de los seres humanos, como son las habilidades que determinan a un buen pensador crítico.

En este sentido, podemos afirmar que si estas habilidades, interpretar, analizar, explicar, son expuestas a los estudiantes a través de situaciones que despierten su interés, sin salirse del ám- 
bito académico, se puede mantener motivados a los jóvenes logrando un mayor porcentaje de mejoría en el desarrollo de las mencionadas habilidades.

Cabe aclarar que los ejercicios requirieron de un tiempo de planeación y direccionamiento para que rindieran los resultados expuestos con anterioridad. Sin embargo, el tiempo que este trabajo de investigación tuvo para implementar las estrategias, se considera poco. No obstante, un proceso de mejoramiento en pensamiento crítico diseñado desde una etapa temprana en los ciclos de educación, podrían ofrecer un avance más significativo en este campo.

Por otra parte, cabe resaltar que los resultados iníciales del diagnóstico son una lamentable muestra del estado en cuanto al pensamiento crítico, en que se encuentran los estudiantes de básica y media vocacional, quienes constantemente tienen que caminar a través de programas pobremente diseñados, con metodologías simplistas que no corresponden a las necesidades de la educación contemporánea, ni mucho menos a las exigencias del MEN, en cuanto al desarrollo de competencias básicas que le permitan a los estudiantes resolver situaciones problemáticas, y por tanto estar mejor condicionados para adaptarse a un mundo cada vez más exigente.

En este sentido, es una conclusión de este trabajo de investigación, que la discusión sobre el estado actual de la educación en Colombia va mucho más allá de la carencia de recursos económicos o de las diferencias socio-culturales.
Una profunda revisión de las formas y los contenidos curriculares, junto con un alto sentido de la responsabilidad de los docentes, es lo que conllevará a un cambio en el paradigma del modelo enseñanza-aprendizaje.

Las pruebas aportadas por este trabajo nos muestran que un proceso bien diseñado, con una estrategia organizada, y a la luz de un modelo pedagógico, puede permitir un avance que subraye en apuntar a la calidad educativa, desde la mejoría de aspectos como la estructura del pensamiento crítico en los niños y jóvenes, que día a día trasiegan por un sistema educativo que dista mucho de sus necesidades esenciales.

De igual forma, cabe resaltar que para efectos de lograr estas mejorías, se requiere una revisión seria y profunda, que vaya desde los procesos de formación de los maestros hasta el diseño de planes de estudios, pasando por las revisiones que deben hacer los entes territoriales para verificar un verdadero crecimiento de los estudiantes en los campos que lo requieren.

\section{Referencias bibliográficas}

Barrel, J. (1999). El aprendizaje basado en problemas - un enfoque investigativo. Buenos Aires: Manantial SRL.

Bisquerra, R. (2009). Metodología de la investigación educativa. Madrid: Editorial La Muralla S.A.

Campos, A. (2007). Pensamiento crítico: técnicas para su desarrollo. Bogotá: Aula Abierta Magisterio. 
Chaffee, J. (1990). Thinking Critically. Boston: Tenth Editions.

Chance, P. (1986). Thinking in the classroom: A survey of programs. New York: Teachers College, Columbia University.

Facione, P. (1990). Critical Thinking: A Statement of Expert Consensus for Purposes of Educational Assessment and Instruction. Recuperado de: https://assessment.trinity. duke.edu/documents/Delphi_Report.pdf

Facione, P. (2007). Pensamiento crítico: ¿Qué es y por qué es importante? Recuperado de: http://www.eduteka.org/pdfdir/PensamientoCriticoFacione.pdf

Fowler, B. (1996). Critical Thinking Definitions. Recuperado de: http://www.Kcmetro. cc.mo.us/longview/ctac/definitions.htm

Galeano, M. (2004). Diseño de proyectos en la investigación cualitativa. Medellín: Fondo editorial Universidad EAFIT.

García, S. (Productor) (2013). Impacto-Guerra entre pandillas. [Archivo de video]. De: https://www.youtube.com/ watch? $\mathrm{v}=\mathrm{Z} 2 \mathrm{HkvjAMMsg}$

Gossaín, J. (28 de febrero de 2014). ¿Por qué es tan mala la educación en Colombia? El Tiempo. Recuperado de: http://www. eltiempo.com/archivo/documento/CMS13570938

Guajardo, G. \& Serrano, F. (2001). Guía técnica para elaborar un ensayo. Querétaro: Universidad Autónoma de Querétaro. Recuperado de: http://www.uaq.mx/FCN/ tutorias/guia_ensayo09.pdf
Hobbes, T. (1651). Leviatán. Recuperado de: http://www.uruguaypiensa.org.uy/imgnoticias/749.pdf

Kant, I. (1803). Pedagogía. Recuperado de: http://dspace.utalca.cl:8888/bibliotecas/ librodot/pedagogia.pdf

Lipman, M. (1997). Pensamiento complejo y educación. Madrid: Ediciones de la Torre.

Lombardero, L. (2015). Trabajar en la era digital: tecnología y competencias para la transformación digital. Madrid: LID Editorial.

Maquiavelo, N. (1513). El príncipe. Recuperado de: http://www.ciudadoriental.com/elprincipe.pdf

Marx, C. (1844). Manuscritos económicos y filosóficos de 1844. Recuperado de: https:// www.marxists.org/espanol/m-e/1840s/ manuscritos/man1.htm\#1-1

Marx, C. (1975). El capital-Tomo I-. México: Siglo XXI Editores.

Merchán, F. (2013, 16 de octubre). El mercado de la fe en Colombia. Supuestos revista económica. Recuperado de: http://revistasupuestos.com/cultura/2015/9/23/el-mercado-de-la-fe-en-colombia

Ministerio de Educación Nacional (2009). Decreto 1290. Recuperado desde: http:// www.mineducacion.gov.co/1621/articles-187765_archivo_pdf_decreto_1290. pdf

Ministerio de Educación Nacional (2010). Orientaciones pedagógicas para la Filosofía en la educación media. Recuperado de: http://www.mineducacion.gov. co/1621/w3-article-241891.html 
Moro, T. (2013). Utopía. Madrid: Ediciones Rialp.

Prieto, G. (Productor) (2013). Especiales Pirry, La enfermedad terminal del sistema de salud. [Archivo de video]. De: https://www. youtube.com/watch?v=tP3-GL2de8w

Reyes, E. (17 de septiembre de 2015). El aborto en Colombia, un camino lleno de baches. El País. Recuperado de: http://internacional.elpais.com/internacional/2015/09/16/ actualidad/1442434449_335332.html

Richard, P. \& Elder, L. (2005). Estándares de competencias para el pensamiento critico. Recuperado de: http://www.eduteka. org/modulos/6/380/2136/1
Rioseco, I. \& Ziliani, M. (1998). Aprendemos y pensamos: lenguaje y comunicación. Santiago de Chile: Editorial Andrés Bello.

Rousseau, J. (1762). Emilio o la educación. Recuperado desde: http://peuma.e.p.f.unblog. fr/files/2012/06/Emilio-ROUSSEAU.pdf

Sartre, J. (1989). El ser y la nada. Madrid: Alianza Editorial.

Solano, V. (Productor) (2013). Documental 9.79 de Victoria Solano. [Archivo de video]. De: https://www.youtube.com/ watch?v=kZWAqS-El_g 